\title{
The effect of agitation state on polyol synthesis of silver nanowire
}

\author{
Amirmostafa Amirjani $^{1} \cdot$ Pirooz Marashi $^{1} \cdot$ Davoud Haghshenas Fatmehsari $^{1}$
}

Received: 14 June 2015/ Accepted: 14 September 2015/Published online: 14 November 2015

(C) The Author(s) 2015. This article is published with open access at Springerlink.com

\begin{abstract}
In the present work, the effect of agitation rate on the growth mechanism of silver nanowires is evaluated during polyol process. It was found that increasing the agitation rate leads to the increase in the oxygen transfer rate which in turn enhances the oxidative etching conditions leading to the formation of a variety of nanostructures (nanoparticles, nanorods and nanowires). In light of the obtained experimental results, it can be stated that agitation is not essential for synthesizing silver nanowires by polyol method and it is possible to obtain uniform nanowires with $\sim 200 \mathrm{~nm}$ diameters in the length of 20-30 microns several microns length in the stagnant condition. By setting the stirring rate at $200 \mathrm{rpm}$, it is possible to reduce the nanowires diameters to $\sim 130 \mathrm{~nm}$ and the obtained nanostructures are still mono-dispersed. This paper provides complete information about the effect of agitation state on the polyol synthesis of silver nanowires which is truly useful for further studies in this case.
\end{abstract}

Keywords Silver nanowire $\cdot$ Polyol synthesis $\cdot$ Agitation condition

Pirooz Marashi

pmarashi@aut.ac.ir

Amirmostafa Amirjani

a.amirjani@merc.ac.ir

1 Department of Mining and Metallurgical Engineering, Amirkabir University of Technology (Tehran Polytechnic), Hafez Ave., P.O. Box 15875-4413, Tehran, Iran

\section{Introduction}

Nanomaterials have versatile applications in electronics, biology and optics which are due to the size-dependent properties of these nanodimensional structures [1]. So, it is possible to achieve desired properties by precise control of the shape and size of the nanocrystals and has interested many researchers during the past decades [2-4]. As chemical synthesis procedures provide better control and monitoring of reaction parameters, accordingly, chemical methods are widely used for nanomaterials fabrication [5].

Polyol process is a well-known method for fabrication of different metal nanocrystals with various shapes including wire, rod, cube and sphere [6-9], and there are many reports regarding parametrical study in polyol process of a specific nanostructure. Noble metal nanocrystals, especially silver, are the center of researchers' attention, and the growth mechanisms and the morphological aspects of silver nanocrystals have been studied by several researchers.

Thermodynamic and kinetic aspects of the synthesis process have the main role in shape evolution of metallic nanocrystals. The latter can be enhanced by the application of heating, stirring and oxidative etching [9]. Multiply twinned particles (MTPs) are thermodynamically stable structures, so it is vital to control the kinetic parameters in synthesis processes to obtain nanostructures with desired shapes. It was found that the synthesis parameters have important roles in the shape transformation of nanostructures [10-12]. Jin et al. [13] synthesized different vaterite crystals by controlling the kinetic and thermodynamic of the growth by changing the precursor molar rations. Cobley et al. [14] controlled the kinetic of the process and achieved desired silver nanostructures by employing a rapid injection of precursors.

An important factor for controlling the kinetics of a synthesis process is the state of agitation in the system. Yan et al. [15] 
used this feature to control the crystal morphology of calcium carbonate. Additionally, Santara and Giri [16] studied the effect of stirring conditions on the synthesis of $\mathrm{TiO}_{2}$. Moreover, the important role of stirring rate on the morphology and optical properties of gold nanorods has been reported by Garcia et al. [17]. It is widely known that the state of agitation affects the crystallization rate and colloids size distribution [18].

A survey of the published reports on the polyol synthesis of silver nanowires provides limited clues on the possible interaction of agitation state of system on the final morphology and size of the obtained nanostructures. To the best of our knowledge, there is no any report regarding the effect of this parameter on the polyol synthesis of silver nanowires. In this study, authors provide complete information about the effect of agitation states on the polyol synthesis of silver nanowires which is truly useful for further studies in this case.

\section{Materials and methods}

\section{Reagents and solutions}

Silver nitrate was purchased from Scharlau; copper chloride dehydrate and ethylene glycol were purchased from Merck; PVP (K-30) was purchased from Sigma-Aldrich (USA), and all the chemicals were used without further purification.

\section{Synthesize procedure}

In a typical synthesis, $10 \mathrm{~mL}$ of $\mathrm{EG}$ was heated to $160{ }^{\circ} \mathrm{C}$ for $60 \mathrm{~min}$. Then, $80 \mu \mathrm{L}$ of $4 \mathrm{mM}$ copper chloride dehydrate was added, and the solution was heated for further $10 \mathrm{~min}$. Then $3 \mathrm{~mL}$ of $114 \mathrm{mM}$ PVP was added to the solution followed by $3 \mathrm{~mL}$ of $94 \mathrm{mM}$ silver nitrate in a fixed rate. During the whole process, the state of agitation was kept constant at three values of 0,200 and $2000 \mathrm{rpm}$ which are specified as A, $\mathrm{B}$ and $\mathrm{C}$ conditions, respectively. The reaction was stopped after approximately one hour. The products were washed three times with acetone and three times with deionized water before further characterization.

\section{Characterization}

Morphological features of the synthesized silver nanostructures were studied using scanning electron microscope (SEM- Philips XL 30) operating at $25 \mathrm{keV}$. Optical characteristics of obtained nanostructures were also studied by UV-Vis spectrometer. An X-ray diffraction unit (Philips PW 1140) was used to study crystal structure of synthesized nanostructures.

\section{Results and discussion}

\section{Characterization of silver nanostructures}

Figure 1 presents the SEM images of obtained silver nanostructures in cases $\mathrm{A}, \mathrm{B}$ and $\mathrm{C}$. The non-homogeneity of products is obvious in case $\mathrm{C}$ where rigorous agitation condition is used. Well-known surface plasmon bands for silver nanowires at $370 \mathrm{~nm}$ and $400 \mathrm{~nm}$ are obvious in Fig. 2 where UV-Vis spectra of three cases are presented. The XRD pattern in Fig. 3 indicates four diffraction peaks are observed at $2 \theta=38.3^{\circ}, 44.4^{\circ}, 64.5^{\circ}$ and $77.4^{\circ}$, corresponding to (111), (200), (220) and (311) planes of the face-centered cubic (fcc) Ag nanostructures (JCPDS File No. 04-0783).

\section{Effect of agitation}

Agitation conditions can effectively influence the rate of a physicochemical process such as nucleation and growth. By comparing the obtained products at different agitation levels (A, B and C), it was observed that there is a 100-nm difference (between A and B cases) in diameter of silver nanowires, which shows the effect of agitation state on growth mechanism of silver nanowires. The diameters of the formed silver nanowires were $\sim 210 \mathrm{~nm}$ and $\sim 130$ for $\mathrm{A}$ and $\mathrm{B}$ conditions, respectively, which shows the homogenizing effect of stirring that hinders the local increase of silver ion in the solution. It is worth to note that a continuous increase in the agitation level will not have a
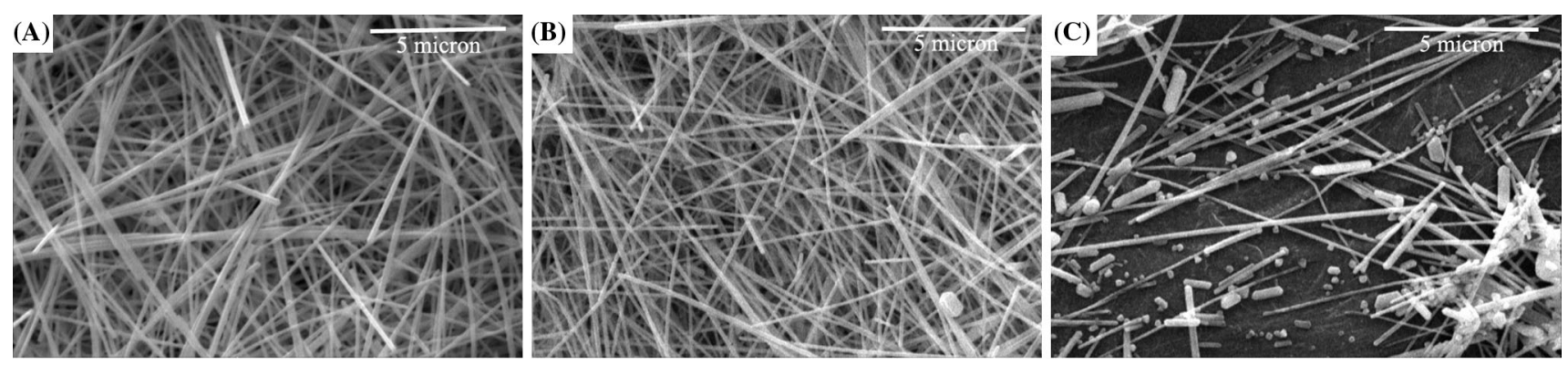

Fig. 1 SEM images of synthesized silver nanostructures obtained without stirring (a), stirring at $200 \mathrm{rpm}(\mathbf{b})$ and stirring at $2000 \mathrm{rpm}(\mathbf{c})$ 


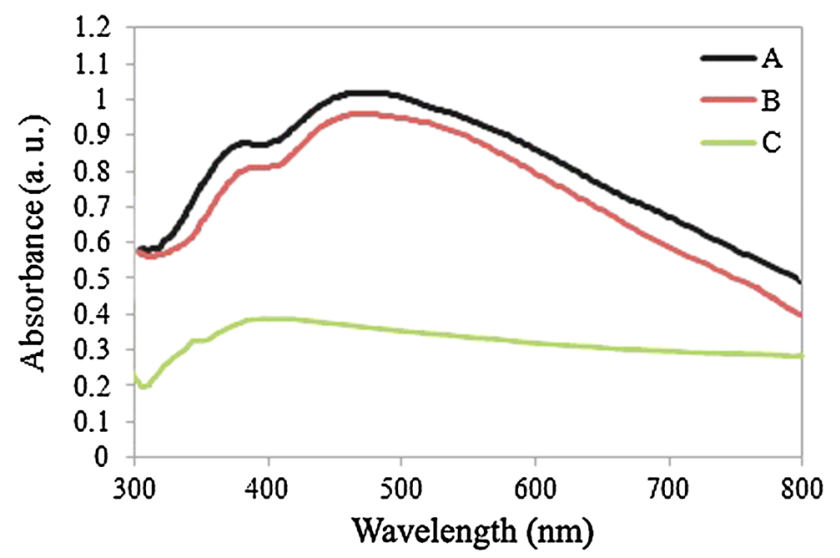

Fig. 2 UV-Vis spectrum of silver nanostructures obtained without stirring (a), stirring at $200 \mathrm{rpm}$ (b) and stirring at $2000 \mathrm{rpm}$ (c)

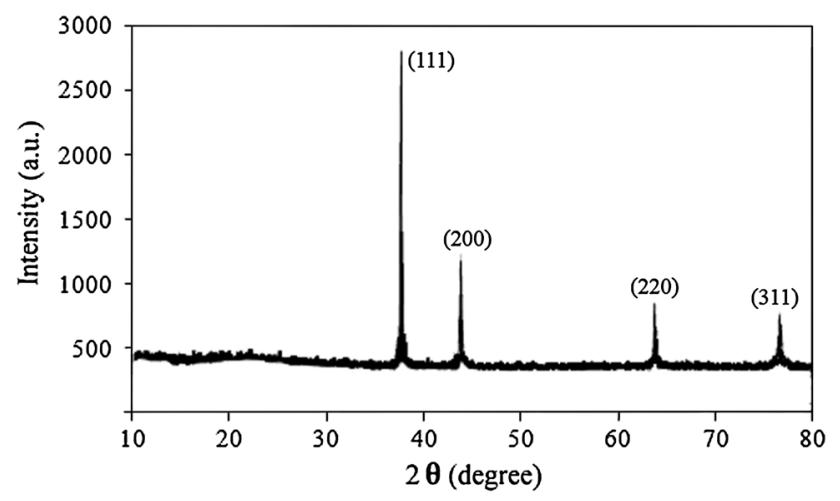

Fig. 3 XRD pattern of silver nanostructures

positive effect on the crystallization and growth conditions of silver nanowires. In case $\mathrm{C}$, where rigorous agitation condition (2000 rpm) was used, non-homogeneous nanostructures were obtained. In this condition, rigorous agitation increases the exposure of nanostructures to oxygen, which remarkably enhances the oxidative etching process and hence the removal of the as-formed multiply twinned particles (MTPs) which are vital for nanowire growth. As seen, obtained nanostructures in case $\mathrm{C}$ are mainly a mixture of null and one-dimensional nanostructures. Another interesting result is the fact that UV-Vis spectrometer is able to predict the obtained nanostructures without any SEM or TEM analysis. In another word, by a close observation in Fig. 2, it can be seen that the spectrum of both A and B cases is similar and also the morphology of these samples is the same as seen in SEM images of Fig. 3. Therefore, UV-Vis spectrometer provides a simple and rapid analysis that makes it easier to find out the morphology of obtained nanostructures which are truly useful in a continuous synthesis procedure. To sum up, based on the above discussions, it can be stated that the rigorous agitation can increase the exposure of nanostructures to oxygen, which remarkably enhances the oxidative etching process.

\section{Conclusions}

In the present study, the effect of agitation rate during polyol synthesis process of Ag nanowires was studied and it was observed that variation in agitation rate, in the range of 0-2000 rpm, not only results in different aspect ratios of one-dimensional nanostructure (nanorod to nanowire) but also significantly stimulates the formation of nanoparticles. Furthermore, this factor can lead to the production of a mixture of one-dimensional and zero-dimensional structures. UV-Vis spectrophotometer was used to predict the obtained nanostructures prior to the morphological analysis using SEM, and it was inferred that UV-Vis spectrophotometer yields reliable data for in situ analysis of colloidal solutions.

Open Access This article is distributed under the terms of the Creative Commons Attribution 4.0 International License (http://crea tivecommons.org/licenses/by/4.0/), which permits unrestricted use, distribution, and reproduction in any medium, provided you give appropriate credit to the original author(s) and the source, provide a link to the Creative Commons license, and indicate if changes were made.

\section{References}

1. El-Sayed, M.A.: Some interesting properties of metals confined in time and nanometer space of different shapes. Acc. Chem. Res. 34, 257-264 (2001)

2. Gholivand, M.B., Malekzadeh, G., Derakhshan, A.A.: Boehmite nano particle modified carbon paste electrode for determination of piroxicam. Sensor Actuat. B Chem. 201, 378-386 (2014)

3. Gholivand, M.B., Jalalvand, A.R., Paimard, G., Goicoechea, H.C., Skov, T., Farhadi, R., Ghobadi, S., Moradi, N., Nasirian, V.: Fabrication of a novel naltrexone biosensor based on a computationally engineered nanobiocomposite. Int. J. Biol. Macromol. 70, 596-605 (2014)

4. Gholivand, M.B., Shamsipur, M., Paimard, G., Feyzi, M., Jafari, F.: Synthesis of $\mathrm{Fe}-\mathrm{Cu} / \mathrm{TiO}_{2}$ nanostructure and its use in construction of a sensitive and selective sensor for metformin determination. Mater. Sci. Eng. C 42, 791-798 (2014)

5. Wiley, B., Sun, Y., Xia, Y.: Synthesis of silver nanostructures with controlled shapes and properties. Acc. Chem. Res. 40, 1067-1076 (2007)

6. Luo, X., Chen, Y., Yang, D., Li, X., Han, Y.: Morphology control of silver nano-crystals through a polyol synthesis. Solid State Sci. 13, 1719-1723 (2011)

7. Ungelenk, J., Speldrich, M., Dronskowski, R., Feldmann, C.: Polyol-mediated low-temperature synthesis of crystalline tungstate nanoparticles MWO4 ( $\mathrm{M}=\mathrm{Mn}, \mathrm{Fe} \mathrm{Co}, \mathrm{Ni}, \mathrm{Cu}, \mathrm{Zn})$. Solid State Sci. 31, 62-69 (2014)

8. Wu, G., Zhou, Y., Gao, X., Shao, Z.: Facile low-temperature polyol process for $\mathrm{LiFePO}_{4}$ nanoplate and carbon nanotube composite. Solid State Sci. 24, 15-20 (2013) 
9. Xia, Y., Xiong, Y., Lim, B., Skrabalak, S.E.: Shape-controlled synthesis of metal nanocrystals: simple chemistry meets complex physics? Angew. Chem. Int. Ed. 48, 60-103 (2009)

10. Amirjani, A., Marashi, P., Fatmehsari, D.H.: Effect of $\mathrm{AgNO}_{3}$ addition rate on aspect ratio of $\mathrm{CuCl}_{2}$-mediated synthesized silver nanowires using response surface methodology. Colloids Surfaces A Physicochem. Eng. Aspects 444, 33-39 (2014)

11. Amirjani, A., Fatmehsari, D.H., Marashi, P.: Interactive effect of agitation rate and oxidative etching on growth mechanisms of silver nanowires during polyol process. J. Exp. Nanosci. (2015). doi:10.1080/17458080.2015.1014872

12. Sadeghpour, S., Amirjani, A., Hafezi, M., Zamanian, A.: Fabrication of a novel nanostructured calcium zirconium silicate scaffolds prepared by a freeze-casting method for bone tissue engineering. Ceram. Int. 40(10B), 16107-16114 (2014)

13. Cobley, C.M., Rycenga, M., Zhou, F., Li, Z.Y., Xia, Y.: Etching and growth: an intertwined pathway to silver nanocrystals with exotic shapes. Angew. Chem. Int. Ed. 48, 4824-4827 (2009)
14. Jin, D., Wang, F., Yue, L.: Phase and morphology evolution of vaterite crystals in water/ethanol binary solvent. Cryst. Res. Technol. 46, 140-144 (2011)

15. Yan, F.W., Zhang, S.F., Guo, C.Y., Zhang, X.H., Chen, G.C., Yan, F., Yuan, G.Q.: Influence of stirring speed on the crystallization of calcium carbonate. Cryst. Res. Technol. 44, 725-728 (2009)

16. Santara, B., Giri, P.K.: Impact of reaction temperature, stirring and cosolvent on the solvothermal synthesis of anatase $\mathrm{TiO}_{2}$ and $\mathrm{TiO}_{2} /$ titanate hybrid nanostructures: elucidating the growth mechanism. Mater. Chem. Phys. 137, 928-936 (2013)

17. García, M.A., Bouzas, V., Carmona, N.: Influence of stirring in the synthesis of gold nanorods. Mater. Chem. Phys. 127, 446-450 (2011)

18. Gavezzotti, A.: Crystal formation and stability: physical principles and molecular simulation. Cryst. Res. Technol. 48, 1-18 (2013) 